\title{
ESTIMACIÓN DE MATERIA SECA Y PARTICIÓN DE ASIMILADOS EN ARBUSTOS DOMINANTES DE DOS MORFOLOGÍAS DIFERENTES DE los Andes Centrales de San Juan, Argentina
}

\author{
DRY MATTER ESTIMATION AND PARTITIONING OF ASSIMILATES IN DOMINANT \\ SHRUBS WITH TWO DIFFERENT MORPHOLOGIES FROM THE HIGH CENTRAL \\ Andes of San Juan, Argentina
}

\author{
Mario Andrés Herrera Moratta ${ }^{1-3 *}$ (D) Ana L. Navas Romero ${ }^{2,3}$, Bárbara Vento ${ }^{3} y$ \\ Eduardo Martínez Carretero ${ }^{3}$ (D)
}

1. Instituto de Biotecnología. Facultad de Ingeniería. Universidad Nacional de San Juan, Argentina 2. Instituto de Ingeniería Química. Facultad de Ingeniería. Universidad Nacional de San Juan, Argentina

3. Instituto Argentino de Investigación en Zonas Áridas. Centro Científico TecnológicoMendoza, Argentina

*maherrera@mendoza-conicet.gob.ar

Citar este artículo HERRERA MORATTA, M. A., A. L. NAVAS ROMERO, B. VENTO \& E. MARTÍNEZ CARRETERO. 2021. Estimación de materia seca y partición de asimilados en arbustos dominantes de dos morfologías diferentes de los Andes Centrales de San Juan, Argentina. Bol. Soc. Argent. Bot. 56: 547-559.

DOI: https://doi. org/10.31055/1851.2372.v56. n4.30589

Recibido: 19 Oct 2020

Aceptado: 28 Sep 2021

Publicado en línea: 15 Nov 2021

Publicado impreso: 20 Dic 2021

Editora: Silvia Lomáscolo (D)

ISSN versión impresa 0373-580X ISSN versión on-line 1851-2372

\section{Summary}

Background and aims: High mountain shrubs play a key role in the maintenance and functioning of ecosystem processes. Nevertheless, studies to understand their morphology and dry matter partitioning are scarce. The goal of this study is to quantify dry matter partitioning and study the morphology for Senecio oreophyton and Baccharis tola ssp. tola in the Central Andes, Argentina. Additionally, allometric equations are proposed.

M\&M: The work was done in the Austral Puna, province of San Juan. A total of $60 \mathrm{~S}$. oreophyton and $B$. tola plants were selected. Biometric variables were measured in each specimen to estimate dry matter. The lateral and top view of each specimen were photographed to determine shrub morphology. Each specimen was harvested and differentiated in stem, leaves and roots.

Results: The geometric shape of $S$. oreophyton was similar to an inverted cone and $B$. tola was similar to a hemisphere shape. In both shrubs, aboveground dry matter was higher than the belowground dry matter. In $B$. tola, similar proportions of dry matter assigned to the stem and leaves were found. Allometric equations were established for each component from direct field measurements.

Conclusions: The dry matter partitioning found for both shrubs would indicate that it is strongly associated with the morphology of the plant, and its strategy for surviving under harsh conditions. This is a contribution to improve the knowledge about morphological characteristics of woody shrubs and their environmental interactions for conservation and management in highlands.

\section{KEY WORDS}

Baccharis tola; conservation; dry matter; highlands; Senecio oreophyton.

\section{RESUMEN}

Introducción y objetivos: Los arbustos de ambientes de alta montaña desempeñan un papel clave en el mantenimiento y el funcionamiento de procesos ecosistémicos. Sin embargo, los estudios para conocer su morfología y su asignación de asimilados son escasos. El objetivo de este trabajo es cuantificar la materia seca y analizar la morfología de Senecio oreophyton y Baccharis tola ssp. tola, en los Andes centrales de Argentina y proponer ecuaciones alométricas.

M\&M: El estudio se realizó en la Puna Austral, provincia de San Juan. Se seleccionaron 60 plantas de $S$. oreophyton y de B. tola. En cada ejemplar se midieron las variables biométricas para estimar la materia seca. Se fotografiaron las vistas lateral y superior para estimar su morfología. Cada ejemplar fue cosechado y diferenciado en tallo, hojas y raíces.

Resultados: La forma geométrica de $S$. oreophyton fue un cono invertido y de $B$. tola una semiesfera. En la materia seca de $B$. tola encontramos valores similares en tallos y hojas, y valores bajos en raíz, mientras que en $S$. oreophyton la materia seca fue mayor en tallos, siguiendo raíz y luego hojas. Se establecieron ecuaciones alométricas para cada componente a partir de mediciones directas de campo.

Conclusiones: La partición de materia seca encontrada para ambos arbustos indicaría una fuerte asociación de la morfología de la planta y su estrategia para sobrevivir en condiciones de altura. Este trabajo es un aporte al conocimiento sobre las características morfológicas de arbustos leñosos y sus interacciones ambientales para la conservación y manejo de ecosistemas de montaña.

\section{Palabras claves}

Ambientes de altura; Baccharis tola; conservación; materia seca; Senecio oreophyton. 


\section{INTRODUCCIÓN}

Los ambientes de alta montaña desempeñan un papel clave en el mantenimiento y el funcionamiento de los ecosistemas, proporcionando un potencial invaluable para rastrear las influencias atmosféricas en la biosfera (Pauli et al., 2005). Estos ambientes se caracterizan por presentar bajas temperaturas, con nevadas periódicas, suelos carentes de materia orgánica y comunidades vegetales con baja cobertura. Solo aquellas plantas con tolerancia a estas condiciones ambientales, como pastos y algunos arbustos, pueden sobrevivir (Squeo et al., 2006). Estos ecosistemas de altura, son altamente sensibles al cambio climático por estar controlados por bajas temperaturas y una considerable amplitud térmica (Pauli et al. 2005). Bajo estas condiciones, los arbustos leñosos son componentes fundamentales que contribuyen al mantenimiento de los procesos ecológicos, la estructura y la biodiversidad de estos ambientes (Martínez Carretero, 1995; Cavieres et al., 2002).

En los Andes centrales de Argentina, los arbustos leñosos dominantes presentan dos morfologías diferentes. Una de las morfologías corresponde a arbustos erectos de copa abierta, y de follaje caducifolio, mientras que la otra morfología corresponde a arbustos de copa cerrada, en contacto con la superficie, y con hojas perennes (arbustos en cojín). Dos especies de arbustos que se corresponden a estas morfologías son Senecio oreophyton J. Rémy, arbusto de copa abierta, y Baccharis tola Phil. ssp. tola, arbusto de copa cerrada (Martínez Carretero, 1995). Ambas especies se encuentran ampliamente diseminadas a lo largo de los altos Andes desde el centro oeste de Argentina hasta el sur de Perú, poseen características morfológicas distintivas y pertenecen a la familia Asteraceae (Herrera Moratta, 2019).

Las estructuras vegetales (hojas, tallo, raíz) son relevantes para entender la interacción planta-ambiente. Dependiendo de las condiciones ambientales dominantes, los organismos asignarán más asimilados a las estructuras que les permitan aumentar la persistencia de la especie (Chapin \& Körner, 1995). El estudio de estas estructuras tiene un alto potencial al brindar información útil sobre los atributos que caracterizan a las especies que dominan en un sistema. En este contexto, la estimación de materia seca es fundamental para conocer cómo responde la estructura de las plantas a las condiciones ambientales (Ward et al., 2016). Para estimar la materia seca aérea y subterránea comúnmente se emplean ecuaciones alométricas. Este tipo de ecuaciones junto con la partición de asimilados aéreos y subterráneos son necesarias para evaluar la sostenibilidad de los ecosistemas y el secuestro de carbono asociado, que resulta crucial para las evaluaciones de acumulación de carbono en ecosistemas de alta montaña (Martínez Carretero et al., 2007; Ward et al., 2016).

El objetivo de este trabajo es determinar la morfología de copa y la partición de materia seca en estructuras aéreas y subterráneas en $S$. oreophyton y $B$. tola ssp. tola, dos arbustos dominantes de ambientes de altura, en los Andes centrales de Argentina. Además, se proponen ecuaciones alométricas para los componentes aéreos y subterráneos de ambas especies.

\section{Materiales y Métodos}

\section{Sitio de Estudio}

El trabajo de campo y la recolección de datos se realizó en el sector noroeste del área privada protegida "Don Carmelo" (3056'24,99" S, 6905'27,6" O), donde la actividad humana está restringida o prácticamente ausente. El área de estudio posee 35.000 ha y se ubica en el centro-oeste de Argentina en la provincia de San Juan. El clima es frío y seco con una temperatura media anual de $9,4{ }^{\circ} \mathrm{C}$ y precipitaciones anuales de $131 \mathrm{~mm}$, que ocurren principalmente durante la temporada de primavera-verano. El mes más frío es julio con una temperatura promedio de 3 ${ }^{\circ} \mathrm{C}$ y el mes más cálido es enero con una temperatura promedio de $19{ }^{\circ} \mathrm{C}$ (Martínez Carretero, 1995; Camarillo-Naranjo et al., 2019). La elevación del área es de 3600 m s.n.m. y se extiende entre las sierras de La Invernada y El Tigre. Esta característica de zona de elevación entre dos cordones montañosos permite la formación de una red de drenaje que culmina en el sur de la reserva aportando los escurrimientos al Río San Juan (Herrera Moratta, 2019) (Fig. 1).

Ambas especies estudiadas están distribuidas y adaptadas a las condiciones ambientales de la provincia fitogeográfica de la Puna, perteneciente al dominio Altoandino (Cabrera, 1968). Según Martínez Carretero (1995) nuestro sitio de estudio pertenece al distrito Cuyano en el sector Austral de la provincia fitogeográfica de la Puna. En esta zona las precipitaciones rondan entre $100-400 \mathrm{~mm}$ al año, con 
M. A. Herrera Moratta et al. - Partición de asimilados en arbustos de altura

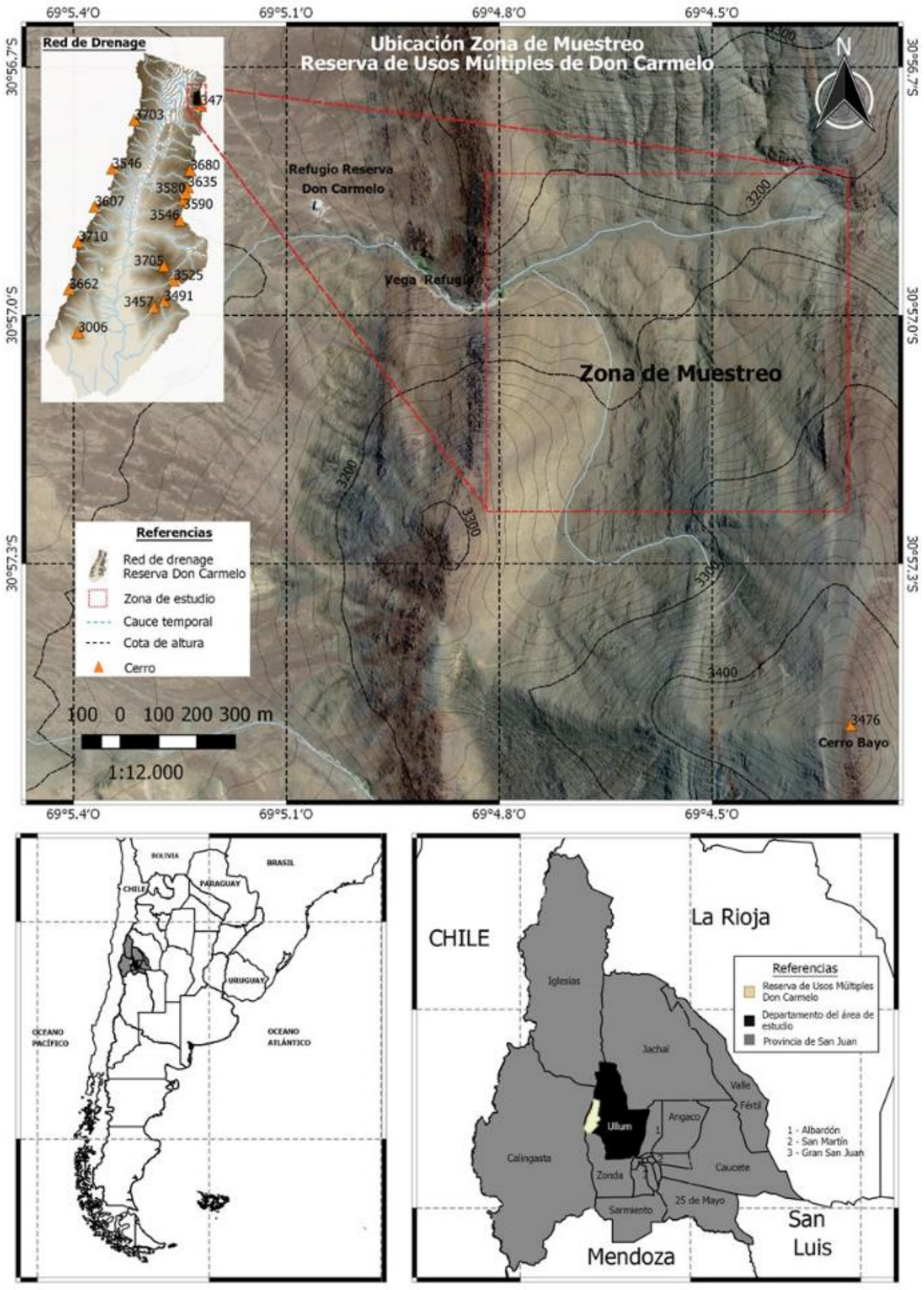

Fig. 1. Área de Estudio: Reserva de usos múltiples de Don Carmelo. 
marcada estacionalidad, con precipitaciones estivales y con sequía invernal (Martínez Carretero, 1995). En general, el área corresponde a una estepa arbustiva ubicada sobre un relieve suavemente ondulado y con presencia de llanuras con vegetación xerofítica corta y valles con sectores de agua permanente (vegas). Las especies más abundantes en la estepa arbustiva son los arbustos Lycium chañar, Artemisia mendozana, Maihueniopsis glomerata, Adesmia aff. horrida, Senecio eriophyton, Baccharis tola ssp. tola y los pastos Stipa speciosa var. breviglumis y Jarava leptostachya. Mientras que en la zona de vegas podemos encontrar especies como Lilaeopsis macloviana, Taraxacum officinale, Nastanthus agglomeratus, Azorella spp.; con una dominancia en laderas rocosas de los arbustos Adesmia pinnifolia y A. triyuga (Márquez, 1998; Lara et al., 2007; Herrera Moratta, 2019).

\section{Selección de especies}

Se seleccionaron dos arbustos perennes, de diferentes morfologías: Baccharis tola Phil. ssp. tola de copa cerrada, crecimiento en cojín y hoja persistente, y Senecio oreophyton J. Rémy, de copa abierta y hoja caduca. Se seleccionaron estas especies ya que ambas codominan en la zona de estudio, pertenecen a la misma familia taxonómica (Asteraceae) y poseen una amplia distribución espacial en las provincias fitogeográficas de la Puna y Altoandina (Martínez Carretero, 1995).

\section{Diseño de muestreo}

Se seleccionaron 60 plantas adultas de Senecio oreophyton y 60 de Baccharis tola en una parcela con un área de $5000 \mathrm{~m}^{2}$, ubicados en las laderas del cerro Bayo (3480 m s.n.m). Dentro de la parcela, se estableció un punto inicial en el extremo oeste, correspondiente a la zona de ingreso a la quebraba del Co. Bayo. Para la selección del primer ejemplar se eligió el individuo de cada especie más cercano al punto de acceso. Luego se seleccionaron sistemáticamente los siguientes ejemplares priorizando aquellos que se encontraban a menor distancia con el ejemplar medido en el punto inicial (elección por proximidad), hasta completar los 60 individuos adultos de cada especie. En cada uno de los individuos se midió la altura total de la planta $(h)$ y el diámetro de copa mayor $(d l)$ y menor $(d 2)$ ambas variables perpendiculares entre sí. Se fotografiaron las vistas lateral y superior de cada individuo, utilizando una cámara Canon T5, para determinar la forma de la copa de los arbustos. Cada imagen fue procesada delimitando el contorno de la planta. Se utilizaron un total de 18 líneas de vista lateral y 18 líneas de vista vertical desde el centro hasta el borde de la planta (separadas $10^{\circ}$ cada una) para determinar la morfología de la copa (Fig. 2). Los datos se ajustaron a una forma geométrica conocida. Las imágenes se procesaron utilizando el software Image J, versión 1.58.

Además, del total de ejemplares seleccionados, 24 individuos de cada especie fueron removidos con sus raíces lo más completas posible, durante la temporada de crecimiento evitando la menor perdida posible de raicillas. Para la elección de los ejemplares a extraer se consideró aquellos individuos que más se acercaron al tamaño medio de la especie en el área de estudio. La recolección de cada ejemplar se realizó de acuerdo con los protocolos establecidos por la Secretaría de Medio Ambiente de San Juan (Disposición SENASA DNPV 4/13). Las muestras se recolectaron de febrero a marzo cuando los arbustos estaban en su máxima expresión vegetativo. Todas las raíces se extrajeron cuidadosamente hasta aproximadamente un metro de profundidad (en suelo con una elevada matriz pedregosa no se encontró material radical por debajo de esta medida) y luego se lavaron por inmersión. Los tallos, hojas y raíces se dividieron cuidadosamente para su secado. Se colocaron muestras de tallo (MSt), hojas (MSh) y raíces ( $\mathrm{MSr} r$ ) en diferentes bolsas de papel y se secaron en estufa a $70^{\circ} \mathrm{C}$ hasta peso constante.

\section{Análisis de los datos}

La independencia estadística y la normalidad de los datos se verificaron mediante la prueba de Kolmogorov-Smirnov (Lilliefors, 1967). La homocedasticidad se verificó mediante la prueba de Levene (Brown and Forsythe, 1974). Los análisis se realizaron considerando un $p<0,05$. Para determinar la relación entre la morfología de cada arbusto y la forma de un cuerpo conocido se realizaron regresiones lineales simples. Los datos de materia seca total (MST), de tallos $(M S t)$, hojas $(M S h)$ y raíz $(M s r)$ junto con el volumen $(V)$ de arbustos se utilizaron para obtener la ecuación alométrica más precisa. Para analizar si existen diferencias estadísticamente significativas en la MSh, MSt y la $M S r$ entre especies se realizó una Anova factorial. Además, se analizó mediante un modelo de regresión múltiple la correlación estadística entre variables biométricas (altura $(h)+$ diámetros $(d 1$ $y d 2)$ ) y la materia seca de cada componente (MST, $M S t, M S h, M S r)$. La significancia y contribución 


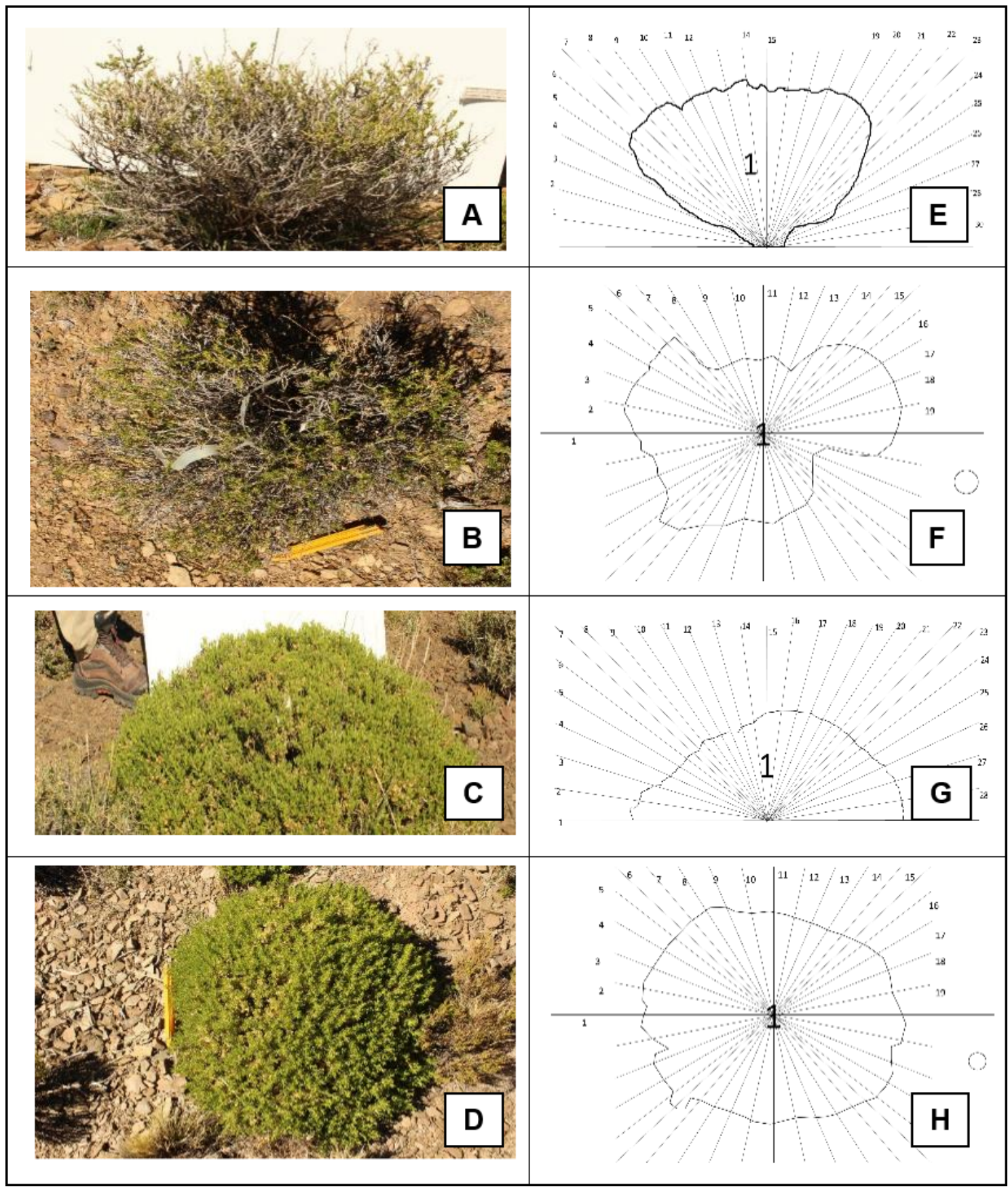

Fig. 2. Vista horizontal y vertical de arbustos leñosos en los Andes centrales. A-B: Senecio oreophyton; C-D: Baccharis tola ssp. tola. Determinación de la morfología de la copa mediante análisis de líneas. E-F: Senecio oreophyton; G-H: Baccharis tola ssp. tola. Separación entre líneas: $10^{\circ}$.

de cada variable morfométrica individualmente se analizó mediante modelos de regresión lineal simple. Los gráficos se realizaron utilizando Sigmaplot v.11 (SigmaPlot, 2008). Los análisis estadísticos se llevaron a cabo utilizando el software SPSS Versión 20 (SPSS Inc., Chicago, IL, EEUU). 


\section{Resultados}

El estudio y análisis de la asociación de la morfología general del arbusto a una forma geométrica específica resultó en Senecio oreophyton similar a un cono invertido (Volumen $=1 / 3^{*} \pi^{*} h^{*} r^{2}$ ) y Baccharis tola a una semiesfera (Volumen $=2 / 3 * \pi^{*} r^{3}$ ), donde $r=$ radio y $h=$ altura. Sin embargo, debido a que no hubo diferencias significativas entre los diámetros de copa menores y mayores, se consideró el diámetro de copa promedio de todos los ejemplares para cada especie. Los valores medios para $S$. oreophyton fueron: volumen $0,029 \pm 0,019 \mathrm{~m}^{3}$, altura total de $0,358 \pm$ $0,082 \mathrm{~m}$ y diámetro de copa de $0,564 \pm 0,140 \mathrm{~m}$. Los valores medios para $B$. tola fueron: volumen $0,146 \pm$ $0,14 \mathrm{~m}^{3}$, altura de $0,213 \pm 0,06 \mathrm{~m}$ y diámetro de copa de $0,859 \pm 0,284 \mathrm{~m}$.

La materia seca total en $S$. oreophyton fue de $700 \pm$ $112 \mathrm{~g}$, distribuidos en: $26 \pm 7 \mathrm{~g}$ para hojas, $564 \pm 103 \mathrm{~g}$ para tallos y $110 \pm 19 \mathrm{~g}$ para raíces. Mientras que en $B$. tola la materia seca total fue de $601 \pm 140 \mathrm{~g}$, con una distribución de $187 \pm 57 \mathrm{~g}$ para hojas, $178 \pm 53 \mathrm{~g}$ para tallos y $50 \pm 23 \mathrm{~g}$ para raíces (Fig. 3). Se detectaron diferencias significativas entre los valores de $M S h$, MSt y MSr para B. tola (Anova, $\mathrm{F}=233,14 ; \mathrm{p}<0,0001$ ) y para $S$. oreophyton (Anova, $\mathrm{F}=769,65 ; \mathrm{p}<0,0001$ ). Hubo diferencias estadísticamente significativas entre especies en los valores de MSh (Anova, $\mathrm{F}=543,73$; $\mathrm{p}<0,0001$ ), de MSt (Anova, $\mathrm{F}=450,51 ; \mathrm{p}<0,0001)$ y en la $\operatorname{MSr}$ (Anova, $\mathrm{F}=114,24 ; \mathrm{p}<0,0001$ ).

La altura fue la variable que mejor estimó la $M S T$, MSt y $M S r$ en ambas especies $\left(\mathrm{R}^{2}>0,90\right)$ (Fig. 4; 5). Además, esta variable resultó ser un buen estimador de MSh de $S$. oreophyton $\left(\mathrm{R}^{2}=0,62\right)$ (Tabla 1; Fig. 4), pero la MSh de $B$. tola fue mejor estimada por el diámetro de copa $\left(\mathrm{R}^{2}=0,92\right)$ (Tabla 2; Fig. 5). En ambas especies, las regresiones múltiples, permitieron obtener ecuaciones que resultaron en un mejor ajuste a los datos que el uso de la formula volumen de cuerpo conocido (Tabla $1 ; 2$ ).

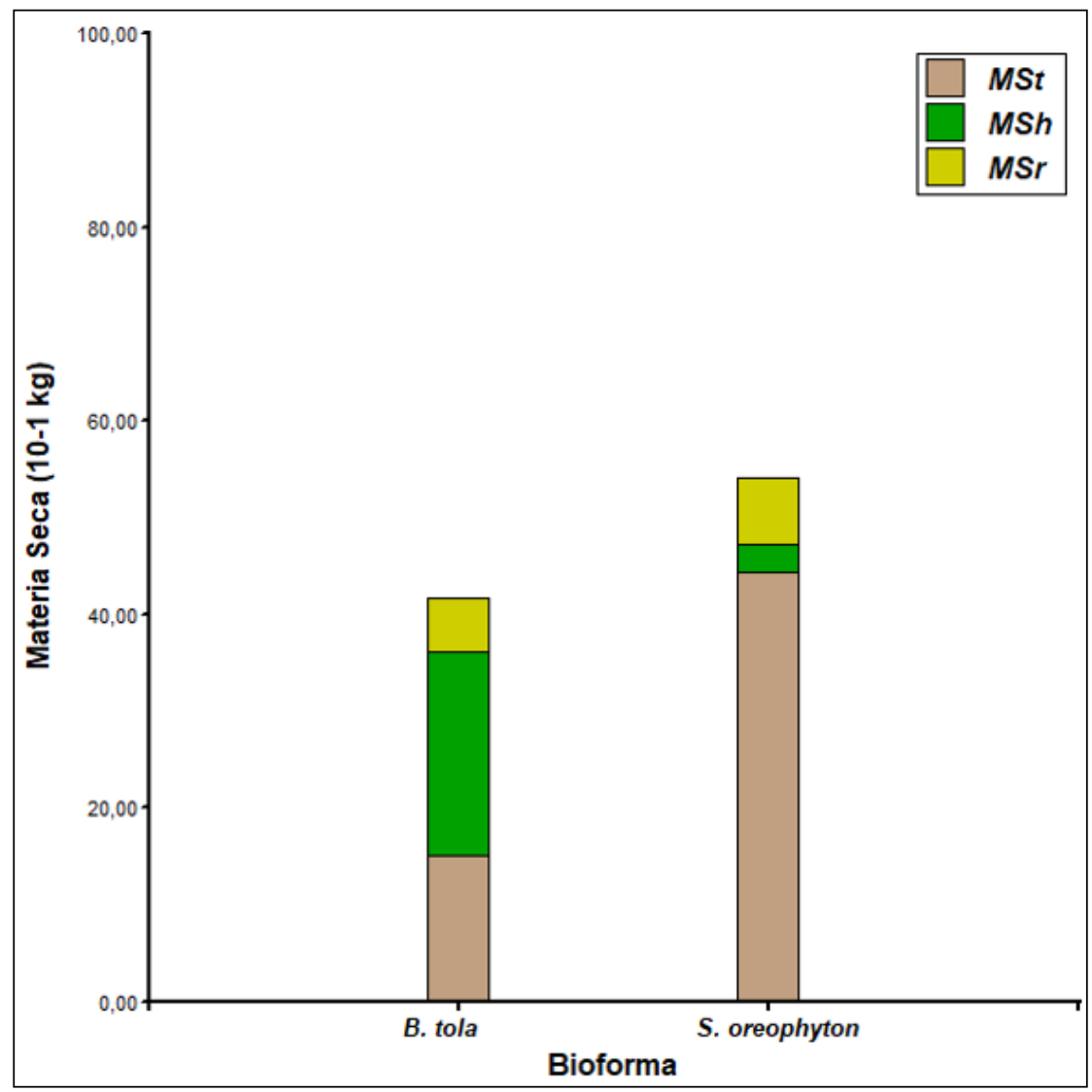

Fig. 3. Distribución de Materia Seca de Tallo, Raíz y Hojas en Senecio oreophyton y Baccharis tola ssp. tola. 
M. A. Herrera Moratta et al. - Partición de asimilados en arbustos de altura

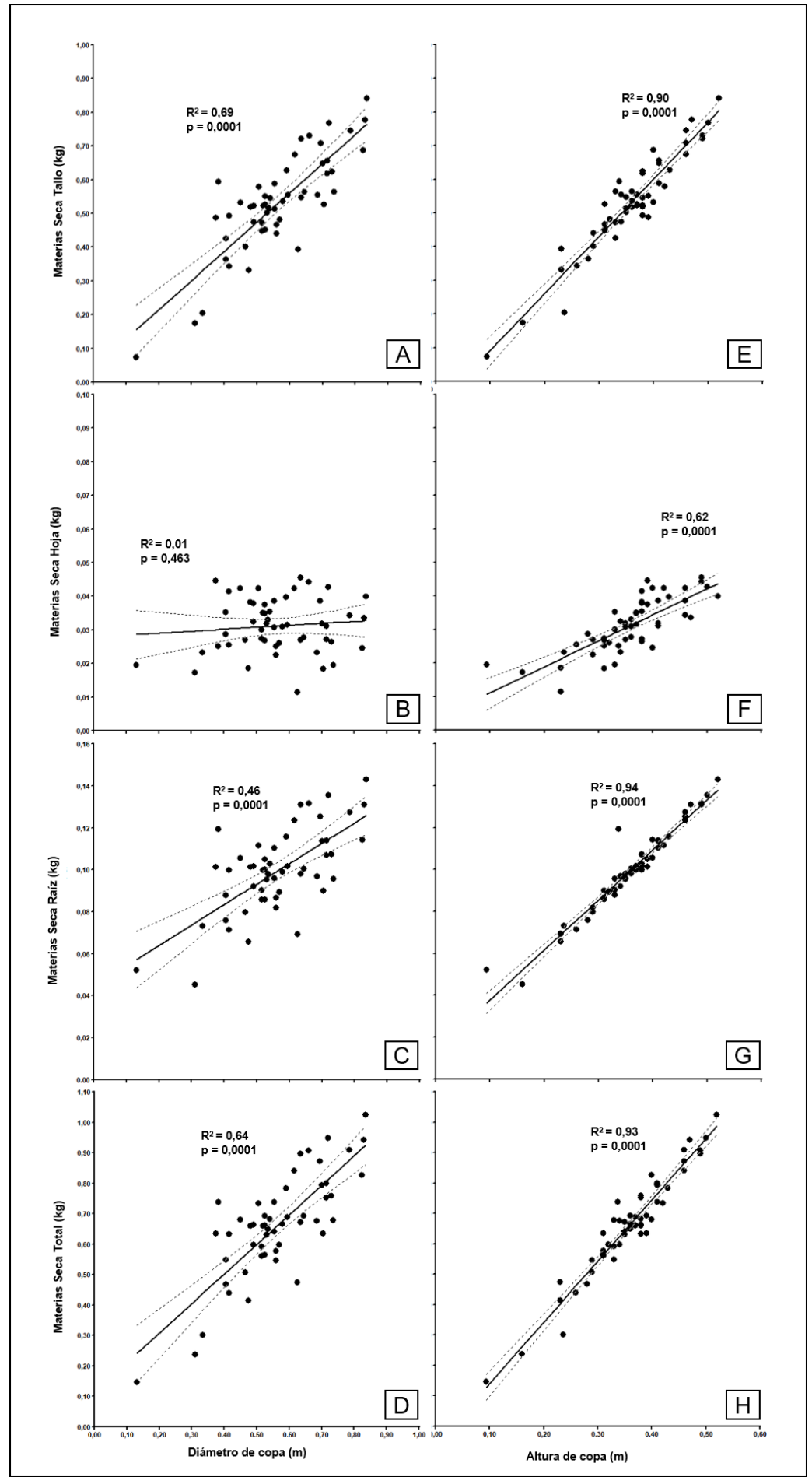

Fig. 4. Modelo de regresión lineal entre la variable morfométrica diámetro $(m)$ y: A: Materia Seca de Tallo, B: Materia Seca de Hojas, C: Materia Seca de Raíz, y D: Materia Seca Total; y entre la variable morfométrica Altura (m), y E: Materia Seca de Tallo, F: Materia Seca de Hojas, G: Materia Seca de Raíz, y H: Materia Seca Total para Senecio oreophyton. 
Bol. Soc. Argent. Bot. 56 (4) 2021

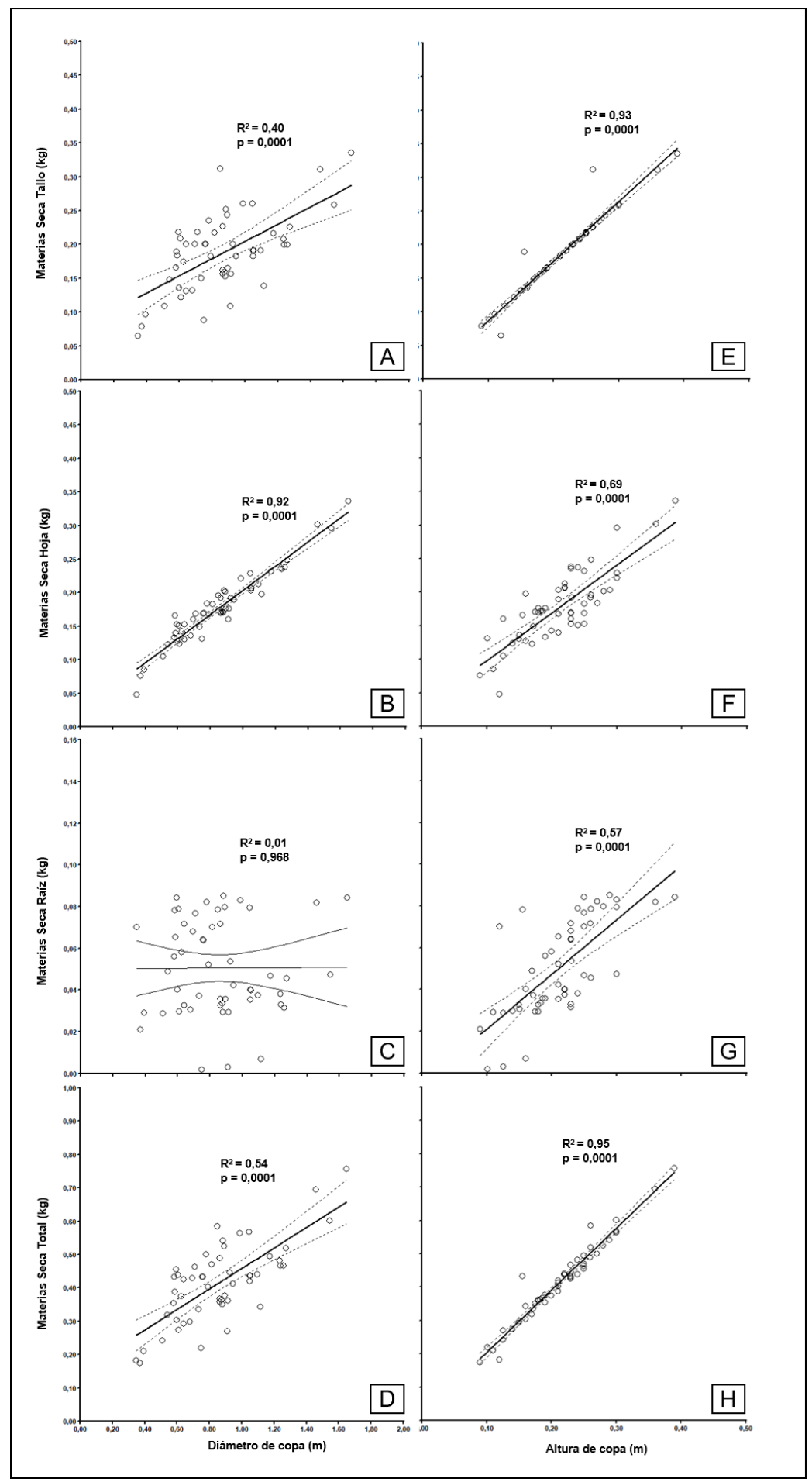

Fig. 5. Modelo de regresión lineal entre la variable morfométrica diámetro $(m)$ y: A: Materia Seca de Tallo, B: Materia Seca de Hojas, C: Materia Seca de Raíz, y D: Materia Seca Total; y entre la variable morfométrica Altura (m), y E: Materia Seca de Tallo, F: Materia Seca de Hojas, G: Materia Seca de Raíz, y H: Materia Seca Total para Baccharis tola ssp. tola. 
Tabla 1. Ecuaciones alométricas para la estimación de Materia Seca de tallos (MSt), hojas (MSh), raíz (MSr), y Materia Seca Total (MST) en Senecio oreophyton con base en medidas morfométricas.

\begin{tabular}{|c|c|c|c|c|c|c|c|c|c|c|c|c|}
\hline \multicolumn{13}{|c|}{ S. oreophyton } \\
\hline \multirow{2}{*}{$\begin{array}{c}\text { Variables } \\
\text { morfométrica }\end{array}$} & Materia Seca & \multicolumn{2}{|c|}{ Total (MST) } & \multicolumn{3}{|c|}{$\begin{array}{c}\text { Materia Seca } \\
\text { Tallo (MSt) }\end{array}$} & \multicolumn{3}{|c|}{$\begin{array}{c}\text { Materia Seca } \\
\text { Hojas (MSh) }\end{array}$} & \multicolumn{3}{|c|}{ Materia Seca Raíz (MSr) } \\
\hline & Ecuación & $\mathbf{R}^{2}$ & $\mathbf{p}$ & Ecuación & $\mathbf{R}^{2}$ & $\mathbf{p}$ & Ecuación & $\mathbf{R}^{2}$ & $\mathbf{p}$ & Ecuación & $\mathbf{R}^{2}$ & $\mathbf{p}$ \\
\hline Altura (A) & $\begin{array}{l}M S T=-0,06 \\
\quad+2,01 \mathrm{~A}\end{array}$ & 0,93 & 0,000 & $\begin{array}{c}\mathrm{MSt}=-0,08 \\
+1,70 \mathrm{~A}\end{array}$ & 0,90 & 0,000 & $\begin{array}{c}M S h=0,003 \\
+0,08 \mathrm{~A}\end{array}$ & 0,62 & 0,000 & $\begin{array}{c}M S r=0,01 \\
+0,24 \mathrm{~A}\end{array}$ & 0,94 & 0,000 \\
\hline Diametro (D) & $\begin{array}{c}M S T=0,11 \\
+0,97 D\end{array}$ & 0,64 & 0,000 & $\begin{array}{l}M S t=0,04 \\
+0,87 \mathrm{D}\end{array}$ & 0,69 & 0,000 & $\begin{array}{c}\text { MSh }=0,03 \\
+0,01 \mathrm{D}\end{array}$ & 0,01 & 0,463 & $\begin{array}{l}M S r=0,04 \\
+0,10 D\end{array}$ & 0,46 & 0,000 \\
\hline Volumen (V) & $\begin{array}{c}M S T=0,43 \\
+7,78 \mathrm{Vol}\end{array}$ & 0,71 & 0,000 & $\begin{array}{l}\text { MSt }=0,33 \\
+6,81 \mathrm{Vol}\end{array}$ & 0,74 & 0,000 & $\begin{array}{l}\text { MSh }=0,03 \\
+0,12 \mathrm{Vol}\end{array}$ & 0,08 & 0,044 & $\begin{array}{l}\mathrm{MSr}=0,07 \\
+0,86 \mathrm{Vol}\end{array}$ & 0,62 & 0,000 \\
\hline $\begin{array}{c}\text { Regresión } \\
\text { múltiple }(A+D)\end{array}$ & $\begin{array}{c}\text { MST }=-0,11 \\
+0,33 \mathrm{D} \\
+1,63 \mathrm{~A}\end{array}$ & 0,97 & 0,000 & $\begin{array}{c}\mathrm{MSt}=-0,13 \\
+0,37 \mathrm{D} \\
+1,27 \mathrm{~A}\end{array}$ & 0,97 & 0,000 & $\begin{array}{c}M S h=0,01 \\
-0,04 \mathrm{D} \\
+0,13 \mathrm{~A}\end{array}$ & 0,96 & 0,000 & $\begin{array}{c}\mathrm{MSr}=0,01 \\
+0,01 \mathrm{D} \\
+0,23 \mathrm{~A}\end{array}$ & 0,94 & 0,000 \\
\hline
\end{tabular}

Tabla 2. Ecuaciones alométricas para la estimación de Materia Seca de tallos (MSt), hojas (MSh), raíz (MSr), y Materia Seca Total (MST) en Baccharis tola ssp. tola con base en medidas morfométricas.

\begin{tabular}{|c|c|c|c|c|c|c|c|c|c|c|c|c|}
\hline \multicolumn{13}{|c|}{ Baccharis tola ssp tola } \\
\hline \multirow{2}{*}{$\begin{array}{c}\text { Variables } \\
\text { morfométrica }\end{array}$} & Materia Seca & \multicolumn{2}{|c|}{ a Total (MST) } & \multicolumn{3}{|c|}{$\begin{array}{l}\text { Materia Seca } \\
\text { Tallo (MSt) }\end{array}$} & \multicolumn{3}{|c|}{$\begin{array}{l}\text { Materia Seca } \\
\text { Hojas (MSh) }\end{array}$} & \multicolumn{3}{|c|}{ Materia Seca Raíz (MSr) } \\
\hline & Ecuación & $\mathbf{R}^{2}$ & $\mathbf{p}$ & Ecuación & $\mathbf{R}^{2}$ & $\mathbf{p}$ & Ecuación & $\mathbf{R}^{2}$ & $\mathbf{p}$ & Ecuación & $\mathbf{R}^{2}$ & $\mathbf{p}$ \\
\hline Altura (A) & $\begin{array}{c}M S T=0,02 \\
+1,86 \mathrm{~A}\end{array}$ & 0,95 & 0,000 & $\begin{array}{c}\text { MSt }= \\
-0,0019+ \\
0,89 \mathrm{~A}\end{array}$ & 0,93 & 0,000 & $\begin{array}{c}\mathrm{MSh}=0,03 \\
+0,71 \mathrm{~A}\end{array}$ & 0,69 & 0,000 & $\begin{array}{c}\mathrm{MSr}=-0,01 \\
+0,26 \mathrm{~A}\end{array}$ & 0,57 & 0,000 \\
\hline Diametro (D) & $\begin{array}{c}M S T=0,15 \\
+0,31 D\end{array}$ & 0,54 & 0,000 & $\begin{array}{c}M S t=0,08 \\
+0,13 D\end{array}$ & 0,40 & 0,000 & $\begin{array}{c}M S h=0,02 \\
+0,18 D\end{array}$ & 0,92 & 0,000 & $\begin{array}{l}M S r=0,05 \\
+0,0046 D\end{array}$ & 0,01 & 0,968 \\
\hline Volumen (V) & $\begin{array}{r}M S T=0,32 \\
+0,67 \mathrm{Vol}\end{array}$ & 0,64 & 0,000 & $\begin{array}{l}\mathrm{MSt}=0,15 \\
+0,29 \mathrm{Vol}\end{array}$ & 0,51 & 0,000 & $\begin{array}{c}M S h=0,13 \\
+0,34 \mathrm{Vol}\end{array}$ & 0,84 & 0,000 & $\begin{array}{l}\mathrm{MSr}=0,04 \\
+0,04 \mathrm{Vol}\end{array}$ & 0,05 & 0,094 \\
\hline $\begin{array}{c}\text { Regresión } \\
\text { múltiple }(A+D)\end{array}$ & $\begin{array}{c}\mathrm{MST}=0,01 \\
+0,06 \mathrm{D} \\
+1,67 \mathrm{~A}\end{array}$ & 0,96 & 0,000 & $\begin{array}{c}\text { MSt }=-0,006 \\
-0,01 \mathrm{D} \\
+0,91 \mathrm{~A}\end{array}$ & 0,93 & 0,000 & $\begin{array}{c}\text { MSh= } \\
-0,001+ \\
0,14 \mathrm{D}+ \\
0,29 \mathrm{~A}\end{array}$ & 0,98 & 0,000 & $\begin{array}{c}M S r=0,01 \\
-0,07 \mathrm{D} \\
+0,48 \mathrm{~A}\end{array}$ & 0,92 & 0,000 \\
\hline
\end{tabular}

\section{Discusión}

Se trabajó con dos morfologías típicas de ambientes de altura: una de copa abierta con morfología similar a un cono invertido, Senecio oreophyton, y otra de copa pulvinada con morfología semiesférica Baccharis tola ssp. tola. Ambas especies poseen alturas menores a $1 \mathrm{~m}$ y una distribución simétrica de asimilados en su copa. Conocer la morfología de un arbusto permite establecer su interacción con el ambiente, por lo tanto, ayuda a comprender como las diferentes arquitecturas de arbustos, permiten diferentes procesos ecológicos en los sistemas naturales (Gautam et al., 2003; Ho et al., 2004; Klich, 2000; Sultan, 2004; Wu \& Hinckley, 2001). Formas de copa semiesférica de arbustos leñosos (forma pulvinada) funcionan como sistemas cerrados, que permiten la acumulación de materia orgánica bajo su dosel, reduciendo la velocidad de la escorrentía del agua, logrando así una mayor retención de la misma en capas superficiales del suelo, y una 
mayor disponibilidad de nutrientes bajo su copa (De Soyza et al., 1997). Mientras que las formas de arbusto de cono invertido funcionan como sistemas abiertos, tienen una mayor exposición de su superficie bajo el dosel a la lluvia, promoviendo que una importante cantidad de agua se pierda debido a la gran superficie de escorrentía, con el consiguiente arrastre y pérdida de materia orgánica y nutrientes (Herrera Moratta, 2019). Por otra parte, en zonas con presencia de viento durante todo el año, se espera que la copa de los arbustos presente signos de influencia del viento en el crecimiento y la distribución de asimilados. Con respecto a la altura de arbustos, se espera una restricción en el tamaño resultando en organismos achaparrados y con crecimiento cercano a la superficie del suelo (Billings y Mooney 1968, Cavieres et al. 1998), y con respecto a la distribución de asimilados, se espera que las mismas presenten variaciones. AlAwadhi et al. (2014), reportó que, para arbustos en el desierto del Sahara, la asimetría encontrada en las canopias responde a la orientación de choque del viento, siendo las secciones a barlovento, zonas con muerte de tejido vegetal, mientras que las secciones a sotavento zonas con mayor crecimiento de tejido vegetal. Sin embargo, en nuestro estudio, en ambos arbustos no observamos la incidencia directa del viento en sus copas. La no incidencia del viento esperada en el eje longitudinal de ambos arbustos, puede ser atribuido a la existencia física de la capa límite, que es la capa de mezcla de aire turbulenta que está en contacto con la superficie de la tierra. Esta capa, por lo general se encuentra entre la superficie del suelo hasta $1 \mathrm{~m}$ de altura, formando una barrera física de aire, que permite evitar el efecto adverso del viento sobre las plantas (Boldes et al., 2003).

Se observaron diferencias en la distribución de la materia seca entre los componentes estructurales analizados. El reparto de la materia seca para S. oreophyton resultó en aproximadamente un $85 \%$ para la parte aérea y un $15 \%$ para la parte subterránea, mientras que para $B$. tola fue aproximadamente del $87,5 \%$ y del $12,5 \%$ para la parte aérea y subterránea respectivamente. En ambas especies, la materia seca subterránea resultó en un menor valor en comparación con la materia seca aérea, y estos valores fueron muy similares, sin embargo, al analizar cada estructura aérea por separado encontramos diferentes estrategias en la asignación de asimilados de hojas y tallo (Fig. 3). Los arbustos con dosel cónico invertido, como S. oreophyton, deben invertir más asimilados en su estructura de soporte, para soportar fuertes vientos y no sufrir desarraigo o exposición de sus raíces (Herrera Moratta, 2019). Esta estrategia de mayor asignación a estructuras de soporte y menor porcentaje de asignación a la parte foliar, probablemente sea consecuencia de la adaptación a las condiciones climáticas frías con un mayor desarrollo de los tallos (Fig. 3); en donde, arbustos de hoja caduca requieren períodos climáticos con condiciones favorables para su crecimiento (Brassard et al., 2009; Ford et al., 2010; Hirose, 2005; Monsi, 1968). Por otra parte, arbustos en cojín y de hoja perenne, como B. tola, necesitan altas asignaciones de asimilados tanto a su sistema de soporte (tallo) como a su masa foliar (hojas) como estrategia para persistir (Fig. 3). Esta asignación de asimilados podría permitir dos mecanismos principales para su adaptación ambiental: uno es la adaptación de la forma de la copa a los fuertes vientos periódicos y a la acumulación estacional de nieve. El otro, es el follaje persistente que mantiene aislado térmicamente el interior de la canopia y conservar temperaturas por encima de $0{ }^{\circ} \mathrm{C}$ en el cuello de la planta durante la temporada de invierno (Billings \& Bliss, 1959; Cavieres et al., 2006; Herrera Moratta, 2019). Además, en estos arbustos pueden permanecer mayores cantidades de carbono en las hojas maduras como reservas para suplir las necesidades de la planta durante la etapa de crecimiento primaveral (Dickmann \& Kozlowski, 1970).

El uso de ecuaciones alométricas permite relacionar variables morfométricas directa mente (altura o diámetro) o indirectamente (ecuación de volumen o de regresión múltiple) con valores de materia seca de arbustos leñosos. En nuestro trabajo, para el arbusto de dosel abierto, S. oreophyton, la altura de la copa fue la mejor variable morfométrica para estimar la materia seca de raíz, tallo y hojas, y para la materia seca de tallo y raíz de B. tola ssp tola. Se han reportado resultados similares para estimar la materia seca en árboles, o en arbustos altos de la Puna boliviana (Halpern et al., 1996; Ketterings et al., 2001). Por otro lado, el diámetro promedio de copa fue la mejor variable para estimar la materia seca foliar en B. tola ssp tola. El diámetro de copa es una variable ampliamente utilizada en trabajos 


\section{A. Herrera Moratta et al. - Partición de asimilados en arbustos de altura}

que utilizan la herramienta GIS (Geographic Information System) para monitorear o conocer la partición de asimilados en los diferentes sistemas (Broadbent et al., 2007; Brown et al., 1989; Rojo et al., 2017). Adicionalmente, el uso de medidas indirectas, como el volumen o ecuaciones derivadas de modelos de regresión múltiple, permiten obtener valores exactos de materia seca de arbustos. Las ecuaciones generadas se pueden extrapolar al rango de especies observadas bajo las mismas condiciones ambientales locales. Sin embargo, es conveniente realizar correcciones de campo cada vez que se quieran utilizar en especies de crecimiento similar, o inclusive en la misma especie. Cuantificar la asignación de asimilados en estructuras de arbustos de los Altos Andes es relevante para estudios posteriores sobre estimaciones de almacenamiento de carbono en estos ambientes naturales, la incidencia del viento en la estructura de la planta, la formación de bancos de semillas, entre otros procesos del ecosistema (Brassard et al., 2009; Flombaum \& Sala, 2007; Martínez Carretero et al., 2007).

\section{Conclusión}

En este trabajo se analizaron las características morfológicas y la distribución de asimilados de dos arbustos dominantes de alta montaña, proponiendo ecuaciones alométricas para cada una de las partes principales de las especies estudiadas.

Los arbustos examinados Senecio oreophyton y Baccharis tola ssp. tola presentan morfologías diferentes que fueron corroboradas a campo. Por un lado $S$. oreophyton resultó similar a un cono invertido con una copa que presenta una gran cantidad de ramillas cubiertas por hojas. Por otra parte, $B$. tola presenta la típica forma de cojín de su copa, similar a una semiesfera con un importante desarrollo foliar. Estas diferencias morfológicas podrían ser el resultado de adaptaciones de estos arbustos a los vientos intensos y persistentes en la zona de estudio.

La distribución de materia seca resultó mayor en la parte aérea que en la subterránea para ambos arbustos con valores similares de materia seca total. Sin embargo, dependiendo de la especie analizada, se registró una mayor cantidad de materia seca para los tallos que para las hojas $(S$. oreophyton) o similar en tallos y hojas (B. tola). Estas diferencias en la distribución de materia seca en las diferentes estructuras vegetativas de la planta podrían relacionarse con las diferentes estrategias de supervivencia a condiciones ambientales de altura.

Las relaciones entre materia seca y las variables morfológicas de las plantas permitieron determinar que la altura de copa resultó ser el mejor estimador de la materia seca total, la materia seca en tallo y en raíz para ambas especies. La altura fue también la mejor estimadora de la materia seca en hojas en $S$. oreophyton, mientras que el diámetro lo fue para B. tola. Las ecuaciones alométricas propuestas resultan de gran utilidad para estimaciones de carbono acumulado en arbustos leñosos de alta montaña.

\section{Contribución de los Autores}

MAHM, ALNR y EMC diseñaron el muestreo, colectaron el material de campo, realizaron el análisis de los datos. BV participó en la redacción y discusión del trabajo. Todos los autores participaron en la escritura del manuscrito.

\section{Agradecimientos}

Agradecemos a Heber Merenda, Andrea Duplancic, Darío Bustamante y Yanina Ripoll por su ayuda en el trabajo de campo. Esta investigación fue apoyada por el Consejo Nacional de Investigaciones Científicas y Tecnológicas (CONICET).

\section{Bibliografía}

AL-AWADHI, J.M. 2014. The effect of a single shrub on wind speed and nabkhas dune development: a case study in Kuwait. Int. J. Geosci. 5: 20. http://dx.doi.org/10.4236/ijg.2014.51004

BILLINGS, W.D., \& BLISS, L.C. 1959. An alpine snowbank environment and its effects on vegetation, plant development, and productivity. Ecol. 40: 388-397. https://doi.org/10.2307/1929755

BOLDES, U., SCARABINO, A., DI LEO, J.M., COLMAN, J., \& GRAVENHORST, G. 2003. Characteristics of some organised structures in the 
turbulent wind above and within a spruce forest from field measurements. J. Wind Eng. Ind. Aerodyn. 91: 1253-1269.

https://doi.org/10.1016/S0167-6105(03)00076-X

BRASSARD, B.W., CHEN, H.Y., \& BERGERON, Y. 2009. Influence of environmental variability on root dynamics in northern forests. Crit. Rev. Plant. Sci. 28: 179-197. https://doi.org/10.1080/07352680902776572

BROADBENT, E.N., ASNER, G.P., PEÑA-CLAROS, M., PALACE, M., \& SORIANO, M. 2008. Spatial partitioning of biomass and diversity in a lowland Bolivian forest: Linking field and remote sensing measurements. For. Ecol. Manage. 255: 2602-2616. https://doi.org/10.1016/j.foreco.2008.01.044

BROWN, S., GILlESPIE, A.J., \& LUGO, A.E. 1989. Biomass estimation methods for tropical forests with applications to forest inventory data. For. Sci. 35: 881-902. https://doi.org/10.1093/forestscience/35.4.881

CAMARILLO-NARANJO, J., ÁLVAREZ-FRANCOSO, J., LIMONES-RODRÍGUEZ, N., PITA-LÓPEZ, M., \& AGUILAR-ALBA, M. 2019. The global climate monitor system: from climate data-handling to knowledge dissemination. Int. J. Dig. Earth. 12: 394-414.

https://doi.org/10.1080/17538947.2018.1429502

CAVIERES, L., ARROYO, M., PEÑAlOZA, A., MOLINA-MONTENEGRO, M., \& TORRES, C. 2002. Nurse effect of Bolax gummifera cushion plants in the alpine vegetation of the Chilean Patagonian Andes. J. Veg. Sci. 13: 547-554.

CAVIERES, L., BADANO, E., SIERRA-ALMEIDA, A., GÓMEZ-GONZÁlEZ, S., \& MOLINAMONTENEGRO, M., 2006. Positive interactions between alpine plant species and the nurse cushion plant Laretia acaulis do not increase with elevation in the Andes of central Chile. New Phytol. 169: 59-69. https://doi.org/10.1111/j.1469-8137.2005.01573.x

CHAPIN, F., \& KÖRNER, C. 1995. Arctic and alpine biodiversity: its patterns, causes and ecosystem consequences. Pp 332. Springer-Verlag Berlin Heidelberg. https://doi.org/10.1007/978-3-642-78966-3

DE SOYZA, A.G., WHITFORD, W.G., MARTÍNEZMEZA, E., \& VAN ZEE, J.W. 1997. Variation in creosotebush (Larrea tridentata) canopy morphology in relation to habitat, soil fertility and associated annual plant communities. Am. Midl. Nat. 137: 13-26. https://doi.org/10.2307/2426751
DICKMANN, D.I., \& KOZLOWSKI, T.T., 1970. Mobilization and incorporation of photoassimilated $14 \mathrm{C}$ by growing vegetative and reproductive tissues of adult Pinus resinosa. Ait. Trees. Plant Physiol. 45: 284-288. https://doi.org/10.1104/pp.45.3.284

FLOMBAUM, P., \& SALA, O.E. 2007. A nondestructive and rapid method to estimate biomass and aboveground net primary production in arid environments. J. Arid Environ. 69: 352-358.

https://doi.org/10.1016/j.jaridenv.2006.09.008

FORD, N.J., LUMB, P.M., \& EKAKA-A, E., 2010. Mathematical modelling of plant species interactions in a harsh climate. J. Comput. Appl. Math. 234, 27322744. https://doi.org/10.1016/j.cam.2010.01.025

FOROUGHBAKHCH, R., REYES, G., ALVARADOVÁZQUEZ, M., HERNÁNDEZ-PIÑERO, J., \& ROCHA-ESTRADA, A. 2005. Use of quantitative methods to determine leaf biomass on 15 woody shrub species in northeastern Mexico. For. Ecol. Manage. 216: 359-366.

https://doi.org/10.1016/j.foreco.2005.05.046

GAUTAM, M.K., MEAD, D.J., CLINTON, P.W., \& CHANG, S.X. 2003. Biomass and morphology of Pinus radiata coarse root components in a subhumid temperate silvopastoral system. For. Ecol. Manage. 177: 387-397. https://doi.org/10.1016/S0378-1127(02)00411-5

HALPERN, C., MILlER, E. \& GEYER, M. 1996. Equations for predicting above-ground biomass of plant species in early successional forests of the western Cascade Range, Oregon. Northwest Sci. 70: 306-320.

HERRERA MORATTA, M. 2019. Interacción planta ambiente en la vegetación de la Puna austral de la Argentina. Tesis Doctoral. Universidad Nacional de Cuyo. Pp 211.

HIROSE, T. 2005. Development of the Monsi-Saeki theory on canopy structure and function. Ann. Bot. 95: 483-494. https://doi.org/10.1093/aob/mci047

HO, M.D., MCCANNON, B.C., \& LYNCH, J.P. 2004. Optimization modeling of plant root architecture for water and phosphorus acquisition. J. Theor. Biol. 226, 331-340. https://doi.org/10.1016/j.jtbi.2003.09.011

KETTERINGS, Q.M., COE, R., VAN NOORDWIJK, M., \& PALM, C.A. 2001. Reducing uncertainty in the use of allometric biomass equations for predicting above-ground tree biomass in mixed secondary forests. For. Ecol. Manage. 146: 199209. 


\section{A. Herrera Moratta et al. - Partición de asimilados en arbustos de altura}

KLICH, M.G. 2000. Leaf variations in Elaeagnus angustifolia related to environmental heterogeneity. Environ. Exp. Bot. 44: 171-183. https://doi.org/10.1016/S0098-8472(00)00056-3

MARTÍNEZ CARRETERO, E. 1995. La Puna Argentina: delimitación general y división en distritos florísticos. Bol. Soc. Argent. Bot. 31, 27-40.

MARTINEZ CARRETERO, E., DALMASSO, A., \& TRIONE, S. 2007. Carbon Storage in Larrea divaricata and L. cuneifolia (Zygophyllaceae) in drylands of central-western Argentina. Arid Land Res. Manage. 21: 273-285. https://doi.org/10.1080/15324980701603409

MONSI, M. 1968. Mathematical models of plant communities. In Functioning of terrestrial ecosystems at the primary production level. Copenhagen, 13: 1-49. Paris: UNESCO

PAULI, H., GOTTFRIED, M., HOHENWALLNER, D., REITER, K., AND GRABHERR, G. 2005. Ecological climate impact research in high mountain environments: GLORIA (global observation research initiative in alpine environments) - its roots, purpose and long-term perspectives. In: Global change and mountain regions, pp. 383-391. Springer, Dordrecht.

https://doi.org/10.1007/1-4020-3508-X_38

ROJO, V., ARZAMENDIA, Y., PÉREZ, $\bar{C} .$, BALDO, J., \& VILÁ, B. 2017. Double sampling methods in biomass estimates of Andean shrubs and tussocks. Rangeland Ecol. Manage. 70: 718-722. https://doi.org/10.1016/j.rama.2017.06.003

SULTAN, S.E. 2004. Promising directions in plant phenotypic plasticity. Perspect. Plant Ecol. Evol. Syst. 6: 227-233.

https://doi.org/10.1078/1433-8319-00082

WARD, A., DARGUSCH, P., GRUSSU, G., \& ROMEO, R. 2016. Using carbon finance to support climate policy objectives in high mountain ecosystems. Clim. Policy. 16: 732-751. https://doi.org/10.1080/14693062.2015.1046413

WU, R., \& HINCKLEY, T.M. 2001. Phenotypic plasticity of sylleptic branching: genetic design of tree architecture. Crit. Rev. Plant Sci. 20: 467-485. https://doi.org/10.1080/07352689.2001.10131827 
